\title{
Lilian Iselin
}

\section{Guerilla-Feldforschung im tibetischen Hochland: Ethische und praktische Herausforderungen}

\begin{abstract}
It is a characteristic of ethnographic field research that it seldom evolves according to plan. Once in the field, researchers must adapt their research designs in response to circumstances and people they encounter. This paper exemplifies this by discussing actual research carried out for the project presented in this paper. The project examines social and spatial transformation in Tibetan nomad communities related to motorized mobility and mobile telecommunication. Geographically, research was located in a contested region of Western China and carried out without official permission. The resulting difficulties and challenges were negotiated by doing guerilla-fieldwork, a term introduced to describe fieldwork pursued without state authorization. This article exposes some of the challenges, both practical and methodological, of guerilla-fieldwork and discusses their implications regarding research ethics. It contributes to current discussions on research ethics and argues that guerilla-fieldwork requires a heightened measure of transparency, openness and reflexivity of the researcher to maintain ethical integrity.
\end{abstract}

Lilian Iselin: Fachbereich Zentralasiatische Kulturwissenschaft, Institut für Religionswissenschaft, Universität Bern. E-mail: lilian.iselin@iash.unibe.ch

\section{Einführung}

Ein weiteres Mal unternahm ich eine Reise in das tibetische Hochland. Dies waren die letzten vier Wochen meiner vier Monate dauernden Feldforschung in der Provinz Sichuan, im Südwesten Chinas. Ich hatte meine Aufenthaltsdauer so eingeteilt, dass ich pro Monat zwei bis drei Wochen auf dem tibetischen Hochland im Norden der Provinz verbrachte und mich danach jeweils wieder in die Provinzhauptstadt zurückzog. Dies war nun meine letzte Möglichkeit, mit tibetischen Nomaden ins Gespräch zu kommen und mit ihnen über ihren Alltag als nomadische Hirten und über den Erwerb und Nutzung von Motorrädern, Autos und Mobiltelefonen zu sprechen. Ich beabsichtigte, Zeit auf Marktplätzen zu verbringen, die von Nomaden aufgesucht werden, um einerseits ihre Mobilitätsmuster 
und ihren Umgang mit mobilen Technologien $\mathrm{zu}$ beobachten und um andererseits weiteres Material über infrastrukturelle Entwicklungen zusammenzutragen.

Mir wurde ein Strich durch die Rechnung gemacht. Meine Forschung im Feld fand ein abruptes Ende, als ich mich nach einer fast zehnstündigen Fahrt ins Hochland zur lokalen Polizeistation begab, um meine Anwesenheit zu melden. Ich bemerkte eine grössere Nervosität als gewohnt. Kurz nachdem ich die Polizeistation verlassen hatte, wurde ich von Beamten angehalten und noch am selben Tag in die Provinzhauptstadt zurück begleitet. Dies war nicht gänzlich unerwartet und nur der Schlusspunkt einer sowohl planerisch als auch emotional anspruchsvollen Zeit im Feld.

Mein Forschungsgebiet gehört $\mathrm{zu}$ der Region, die seit drei Jahren immer wieder durch Akte der Selbstverbrennung von Tibetern, mehrheitlich aus dem klösterlichen Umfeld, in die internationalen Medien gelangt ist. Da erstaunt es nicht, dass das Gebiet sowie meine Forschung, die sich mit Mobilität und Mobiltelefonie befasst, als ,politisch heikel' gelten. Mir gelang es nach wiederholten Bemühungen nicht, eine Forschungsbewilligung $\mathrm{zu}$ erwirken. ${ }^{1}$ Das politisch angespannte Klima in tibetischen Regionen der Volksrepublik China hatte sich 2012 durch den bevorstehenden Regierungswechsel im Spätherbst verstärkt. Dies und die anhaltenden Aktionen des Widerstands in tibetischen Regionen liessen vermuten, dass sich die Lage in nächster Zukunft nicht wesentlich entspannen würde. Ich hatte die Wahl, meine Forschung auf eine unbestimmte Zukunft zu verschieben oder flexibel auf veränderte Umstände zu reagieren, um meine Feldforschung überhaupt antreten zu können. Wie viele andere Forschende vor mir entschloss ich mich, als Reisende statt als Forschende ins Zielgebiet zu gehen. Damit begab ich mich in eine Zone, die befrachtet ist mit einer Reihe ethischer Fragen in Bezug auf den Prozess der Forschung. Selten wird explizit über und aus dieser Grauzone der Feldforschung berichtet. Ich möchte sie hier unter dem Begriff „Guerilla-Feldforschung“2 thematisieren.

Guerilla-Feldforschung bezieht sich auf das Unordentliche, Kontroverse und Ausserordentliche einer Feldforschung, die im Graubereich eines staatlich nicht autorisierten Projekts stattfindet. Ich möchte meine eigene Erfahrung offenlegen und aufzeigen, wie ich mit ethischen und anderen Konflikten und Herausforderungen umgegangen bin. Dadurch möchte ich zugleich die Chancen und Limitati-

\footnotetext{
1 Über den langen und komplizierten Prozess durch die Administration der Volksrepublik China, der im besten Fall schlussendlich zu einer Forschungsbewilligung führt, hat Candice Cornet (2010) ausführlich berichtet.

2 Ich lehne mich hier an einen Begriff von Thomas Gold an, der im Kontext der Feldforschung in China von „guerilla interviewing“ als eine Methode, die Limitationen staatlich kontrollierter Forschung zu umgehen, vorgeschlagen hat (1989).
} 
onen einer Guerilla-Forschung problematisieren. Ich beginne damit, dass ich den Kontext meines Dissertationsprojekts erläutere und dadurch begründe, weshalb Ethnographie als Methode am geeignetsten ist, die Forschungsfragen des Projekts zu untersuchen. Im Weiteren werde ich die Forschungsmethode und ihren ethischen Anspruch erläutern. Eine Darstellung des Vorgehens im konkreten Forschungskontext wird aufzeigen, welche Schwierigkeiten sich gestellt haben und wie damit umgegangen wurde. Lösungsansätze werden kritisch thematisiert und Fragen der Transparenz und Forschungsethik zur Diskussion gestellt.

Mit meinem Aufsatz möchte ich mich einem wachsenden Korpus an Veröffentlichungen anschliessen ${ }^{3}$, welcher die oft nicht lineare, frustrierende, und komplexe Erfahrung der Feldforschung zum Thema macht. Ähnlich wie Emily Yeh (2006), möchte ich mein eigenes Erleben zum Ausgangspunkt nehmen, um zu zeigen, wie politische Einschränkungen das Forschungsdesign definieren, und wie dies manchmal zu unerwarteten Resultaten führen kann. Ich lege den Fokus auf einen selten besprochenen Aspekt, nämlich, wie im konkreten Fall der Guerilla-Feldforschung mit dem ethischen Anspruch umzugehen ist.

\section{Die Forschungsfrage und der Forschungskontext}

Das tibetische Hochplateau gehört $\mathrm{zu}$ einer wirtschaftlichen Randregion des sich rasant entwickelnden Chinas. Es ist zudem von Menschen einer ethnischen Gruppe bewohnt, die in China als ,nationale Minderheit' klassifiziert ist. Beide Einteilungen machen das tibetische Hochland zum Empfänger von Entwicklungshilfe einerseits und Modernisierungsbestrebungen der Regierung der Volksrepublik China andererseits. Beides äussert sich in Politiken, die zum Teil koloniale Züge tragen. ${ }^{4}$ Diese hegemonialen Konstellationen kommen in Diskursen zur Infrastrukturentwicklung im tibetischen Hochland zum Ausdruck. Infrastrukturbauten werden einerseits als Zeichen einer erstrebenswerten Modernisierung und als Grundlage für wirtschaftliches Wachstum durch den Staat vorangetrieben $^{5}$ und sichern andererseits staatlichen Zugang und Kontrolle. Deshalb hat Infrastrukturentwicklung in China eine geo- und machtpolitische Komponente, welche zum Teil kritisch diskutiert wird 6 .

3 Siehe z.B. Billo und Hiemstra 2012; Bonnin 2010; Cornet 2010; Heimer und Thøgersen 2006; Wood 2006.

4 Für eine kritische Diskussion des Begriffs siehe Sautman 2006.

5 Démurger 2001; Wang 2007.

6 Arya 2011; Flower 2004; Garver 2006. 
Die Makroperspektive sowohl einer Geo- als auch einer staatlichen Entwicklungspolitik ist allerdings nur einer der möglichen Aspekte, wie die Materialität der Infrastrukturbauten betrachtet werden kann. Michel de Certeau (1988) machte darauf aufmerksam, dass eine totalisierende Sicht durch die tägliche Praxis derjenigen subvertiert wird, die sich in den Räumen bewegen und sich diese zu Nutze machen. Entsprechend will das vorliegende Forschungsprojekt das Alltägliche aufzuzeigen. Es befasst sich damit, wie tibetische Nomaden Strasseninfrastruktur und Telefontürme und deren Reichweiten in ihre Lebenswelt auf dem Weideland integrieren. Es untersucht, wie mobile Technologien, deren Nutzung durch die Infrastruktur ermöglicht oder erleichtert wird, in der alltäglichen Praxis der Nomaden eingesetzt werden und welche Veränderungen in räumlichen Zuteilungen und sozialen Beziehungen dadurch stattfinden. Statt die Sicht der Entwicklungsplaner, also des Staates, in den Fokus zu rücken, werden die Menschen, die die Infrastrukturen nutzen, untersucht.

Tibetische Nomaden des Nordens der Provinz Sichuan haben in den 1990er Jahren begonnen, sich Motorräder anzuschaffen und seither hat die Motorisierungsrate tibetischer Haushalte kontinuierlich zugenommen. Eine Zunahme ländlichen Strassenbaus hat zusätzlich die Einbindung der Nomaden in die vom Staat geförderte und geforderte Marktwirtschaft und Prozesse der Urbanisierung beschleunigt. Praktisch zeitgleich zu der Entwicklung der zunehmenden Motorisierung nomadischer Haushalte entwickelte sich die Mobiltelefonie in China und weltweit zu einem Massenprodukt, und der entsprechende Infrastrukturausbau des Mobilfunknetz wurde staatlich vorangetrieben ${ }^{7}$. Im Forschungsgebiet im Norden von Sichuan, in welchem bis Ende der 1990er Jahre kein durchgehendes Telefonnetz mit Festnetzanschlüssen für Siedlungen bestand, ging man direkt dazu über, ein Mobilfunknetz einzurichten, das man seit seinen Anfängen kontinuierlich ausbaute. Erst der Mobilfunk gab den verstreut lebenden und mobilen Nomaden Zugang zur Telefonie.

Der Ausbau der Infrastruktur erfolgte zum grossen Teil auf staatliche Initiative. Der Gebrauch von Motorfahrzeugen und Mobiltelefonen ist aber auf der Ebene ihrer Besitzer zu untersuchen. Die Annahme dieser Studie ist, dass die Nutzung beider mobiler Technologien Auswirkungen auf die Bewegungs- und Handlungsräume der Nomaden hat. Beispielsweise lässt sich feststellen, dass Besitzer eines Mobiltelefons die Reichweite des Signals eines Telefonturms kennen und sich in ihrem Handeln und ihren Bewegungen danach ausrichten. Telefontürme schaffen Bezugsfelder, innerhalb derer telefonische Erreichbarkeit gewährleistet ist. Wer motorisiert ist, sucht sich andere Routen aus, als wenn er

7 Schüller, Schüler-Zhou und Peterskovsky 2010. 
zu Fuss oder zu Pferd unterwegs wäre. Die gebauten Strassen definieren Richtungen, die ins Land eingeschrieben werden. Dieses Netzwerk von Infrastrukturbauten überlagert ein Gebiet, das von Menschen bewohnt wird, deren Lebensweise sich durch Mobilität auszeichnet. Die Frage stellt sich, wie tibetische Nomaden die Infrastruktur und die Möglichkeiten, die ihnen diese eröffnet, in ihren Alltag einbetten, und wie sich veränderte Mobilität im alltäglichen Geschehen abbildet. Das Forschungsprojekt will untersuchen, welche Verschiebungen sich im räumlichen Alltagshandeln ergeben und welche Auswirkungen dies auf die sozialen Beziehungen in Gemeinschaften tibetischer Nomaden hat.

Diesen Fragen ging ich mit dem Instrumentarium der qualitativen Sozialforschung nach. Durch teilnehmende und nicht-teilnehmende Beobachtung sowie durch freie oder semi-strukturierte Gesprächsführung untersuchte ich, wie die Infrastruktur genutzt wird, wie sie im Alltag der Nomaden Bedeutung erlangt, und wie Erwerb und Nutzung von mobilen Technologien die Mobilität der Nomaden verändert. Dabei war vorgesehen, ihre alltägliche Praxis zu untersuchen und ihren Lebensort, die Weidegebiete, zum Ausgangspunkt ihrer Mobilitäten zu nehmen. Da nomadischer Pastoralismus bei den Tibetern auf der Basis von Familieneinheiten erfolgt, bezog ich mich methodisch auf diese Einheit. Ich hatte geplant, mich einige Wochen in nomadischen Gebieten aufzuhalten, dabei Familien für mehrere Tage in den Weidegebieten $\mathrm{zu}$ besuchen, um sie in ihrem Alltag zu beobachten und mit ihnen Gespräche über ihren Lebensalltag, Umgang mit motorisierten Fahrzeugen und Mobiltelefonie zu führen. Bevor ich meine methodischen Pläne umsetzen konnte, wurde ich von der vorgängig beschriebenen, komplexen Realität im Forschungsgebiet eingeholt und musste mich mit viel Flexibilität auf veränderte Umstände einstellen.

\section{Guerilla-Feldforschung und Forschungsethik}

Feldforschung entwickelt sich selten so, wie man es sich vorher vorstellt und im Forschungsplan beschreibt. Ein hohes Mass an Flexibilität, Reflexivität, Spontaneität und Offenheit dem Unternehmen Feldforschung gegenüber ist ein Grundanspruch an die ethnographischen Arbeitsweise. ${ }^{8}$ Dies wurde in dem hier beschriebenen Projekt insbesondere dadurch zwingend, da ich mich trotz ausstehender Forschungsbewilligung entschieden hatte, meine Feldforschung durchzuführen. Ich musste dadurch flexibel auf veränderte Umstände reagieren, auch um sicherzustellen, dass in der Durchführung dieser Guerilla-Feldforschung, wie ich sie nenne, die Forschungsethik aufrecht erhalten werden konnte.

8 Siehe z.B. Davies 1999; Fabian 2001; Geertz 1973. 
Ethnographische Forschungsethik beinhaltet für mich drei wesentliche Punkte: Erstens soll den an der Forschung beteiligten Personen kein Schaden erwachsen. Zweitens sind die Forschungsabsichten offenzulegen und die Bereitschaft der Betroffenen, sich an der Forschung zu beteiligen, sicherzustellen. Drittens muss die eigene Subjektposition unter Bezugnahme allfälliger Machtgefälle reflektiert werden. Ich lehne mich in der Gewichtung der Punkte an die Leitlinien der American Anthropological Association an, wie sie im Statement on Ethics: Principles of Professional Responsibility ${ }^{9}$ definiert sind. In der Praxis meiner Feldforschung ergaben sich besondere Herausforderungen bei der Umsetzung, die im Folgenden ausgeführt werden sollen.

Guerilla-Feldforschung führt $\mathrm{zu}$ Dilemmas bezüglich einiger dieser Prinzipien. Beispielsweise halten sie fest, dass zu jedem Forschungsvorhaben die Zustimmung der Betroffenen einzuholen ist, bzw. dass sie darüber informiert sein sollen, wozu ihre Aussagen und Informationen benutzt werden und was das Ziel der Forschung ist. Guerilla-Feldforschung bedeutete nun aber, dass ich nur beschränkt offen über meine Absichten reden konnte, insbesondere um dem ersten Punkt, dass niemandem Schaden erwachsen soll, Rechnung zu tragen. Ich betrieb also zu einem gewissen Grad verdeckte Forschung, welche in der Literatur zu qualitativer Forschung kritisch diskutiert wird. ${ }^{10}$ Weitere ethische Dilemmas betrafen meine Subjektposition. Da ich nicht offen als Forschende auftreten konnte, bediente ich mich verschiedener Rollen, die meinen Gesprächspartnern zum Teil aus früheren Aufenthalten im Forschungsgebiet bekannt waren. Dies stellt Anforderungen an die Reflexion von Gesprächs- und Beobachtungssituationen im Feld und fordert kritisches Abwägen, ob und welche Daten benutzt werden können. Guerilla-Feldforschung hatte auch zur Folge, dass ich nicht nur mich, sondern potentiell auch meine Gesprächspartner, in eine Situation brachte, die vom bestehenden Machtapparat nicht sanktioniert war und deshalb das Potential in sich trug, Konsequenzen für die Involvierten nach sich zu ziehen. Diesem für mich gewichtigsten Punkt gab ich in der Ausführung der Feldforschung am meisten Aufmerksamkeit.

In der praktischen Umsetzung der Feldforschung entwickelte ich Vorgehensweisen, die manchmal chaotisch und anfangs wenig reflektiert waren. Sie entstanden zum Teil ad hoc in der täglichen Interaktion mit den Menschen, deren Alltagspraktiken meinen Untersuchungsgegenstand bildeten. Der Minimalanspruch meiner Forschungsethik war, dass ich keine der Personen, mit denen ich in Kontakt trat, in irgendeiner Art kompromittierte. Diese Anforderung beruft

9 Association 2012.

10 Bryman 2001: 292-293; Davies 1999: 53-61. 
sich auf den ersten Punkt der Statement on Ethics, welcher festhält, dass nichts und niemandem durch die Forschung oder die Anwesenheit des Forschenden Schaden erwachsen darf, ${ }^{11}$ und wird als Prinzip des „Do no harm“ beschrieben. In der Rolle der Reisenden und als Besucherin von Freunden und Bekannten stellte ich mir konstant die Frage, wie dieser Anspruch aufrechterhalten werden kann, und entwickelte hierzu Strategien, die ich im Folgenden ausführe.

Meine Begegnungen und Gespräche mit Menschen im Forschungsgebiet blieben informeller Natur. In Anwendung des Prinzips „Do no harm“ steuerte ich den Gesprächsinhalt wenn möglich und nötig so, dass meine Gesprächspartner - sollten sie von den Behörden zu den Inhalten befragt werden - Auskunft geben konnten, ohne sich selber in eine schwierige Lage zu bringen. Gesprächsinhalte blieben deshalb zwangsläufig manchmal an der Oberfläche des Alltäglichen und Beschreibenden. Auf Leitfadengespräche mit Angehörigen von nomadischen Familien, die in schriftlichem oder im Audioformat aufgezeichnet wurden, verzichtete ich fast vollständig. Im Forschungsgebiet agierte ich äusserst vorsichtig und suchte dieselben Personen nicht gehäuft auf. Ich strebte an, mich wie eine Reisende zu verhalten, die Bekannte und Freunde besucht.

Der oben beschriebene Umgang mit den Menschen, denen ich begegnete, hatte Konsequenzen in Bezug auf den ethischen Anspruch der Offenlegung meiner Forschungsabsichten. Um meine Gesprächspartner zu schützen, musste ich betreffend der Gründe meiner Anwesenheit von Fall zu Fall abschätzen, wie diese zu formulieren seien, und blieb manchmal vage. In Fällen, in denen ich meine Absichten als Forschende nicht genügend deklarieren konnte, hat das zur Folge, dass ich Gesprächsinhalte nur nach sorgfältigem Abwägen des Kontexts für die Forschung verwenden kann und manchmal, um einem ethischen Standard zu genügen, auf deren Verwendung verzichten muss. In meiner Rolle als Reisende unternahm ich zudem nicht-teilnehmende Beobachtungen. Sie erfolgten in erster Linie in öffentlich zugänglichen Räumen und mit viel Diskretion in meinem Netzwerk von Freunden und Bekannten. Auch dieses Vorgehen verhinderte eine völlige Offenlegung meiner Absichten und ähnelt zum Teil verdeckter Forschung.

In Bezug auf den dritten Punkt, der Rolle der Ethnographin und ihre Subjektposition, ergab sich das Dilemma, dass ich aufgrund fehlender Forschungsbewilligung mein persönliches Netzwerk von Freunden und Bekannten beanspruchte. Man könnte argumentieren, dass dies ideale Voraussetzungen sind, weil bereits eine Vertrauensbasis besteht, und Freunde am ehesten über die Forschungsabsicht der Ethnographin informiert sind. Dies lässt aber ausser Acht, dass Freundschaften aus Beziehungen des Gebens und Nehmens bestehen. Für einige meiner

11 Siehe auch Yeh 2006: 108-109. 
Gesprächspartner mag ein moralischer Druck bestanden haben, mir zu helfen. Zusätzlich ist zu bedenken, dass auch in Freundschaften und freundschaftlichen Beziehungen Machtgefälle bestehen können, insbesondere wenn die Forschende als Ausländerin einen anderen Status und andere Möglichkeiten zur Lebensgestaltung hat. ${ }^{12}$

Um dieses Beziehungsnetz nicht überzustrapazieren, bin ich auf eine indirekte Forschungsmethode ausgewichen. Ich suchte ausserhalb des Forschungsgebiets Menschen aus nomadischen Familien, die beispielsweise in der Stadt die Universität besuchen oder dort arbeiten, und befragte sie zu ihren Familien. In der Anonymität einer chinesischen Grossstadt entzog ich sie der Beobachtung des Staates und konnte erklären, welche Forschung ich verfolgte und wofür die Gespräche verwendet würden. Meinen Gesprächspartnern habe ich persönliche Anonymität zugesichert, sowie die Unkenntlichmachung von Orten und Personen, die in den Gesprächen erwähnt wurden. Ich konnte damit einem ethischen Anspruch des Quellenschutzes genügen. Dies ging allerdings auf Kosten der Nähe zum Forschungsfeld. Meine Gesprächspartner wurden zu Übersetzern der Vorgänge und der Lebenswelt der Nomaden. Dadurch gingen Unmittelbarkeit und Tiefe in Bezug auf das Datenmaterial verloren.

In einem komplexen Forschungskontext habe ich mich im Prozess des ethnographischen Arbeitens vorgetastet und Strategien angewandt, die oft aus der Notwendigkeit geboren waren. Ich habe während der Forschung immer wieder den Ansatz „Do no harm“ reflektiert sowie meine eigene Subjektposition in Bezug auf die Menschen, mit denen ich in Beziehung trat. Die daraus resultierenden Daten sind anders ausgefallen als ursprünglich geplant, und es stellen sich mir in der Auswertung einige Probleme, die ich im nächsten Teil diskutieren möchte.

\section{Nach der Guerilla-Feldforschung: Fragen zur Datenmenge, zum Forschungskontext, zum reflexiven Umgang mit und zum ethischen Anspruch an die Daten}

Ethnographie ist eine induktive und intuitive Methode. Beobachtungen, Gespräche, sowie Informationen aus Dokumenten wie Zeitungen, Webseiten etc. werden trianguliert. Eindrücke, Gehörtes, Erlebtes und Gelesenes werden gegeneinander abgeglichen und auf Gemeinsamkeiten und Unterschiede untersucht. Mit einer

12 Siehe Bonnin 2010: 188. 
genügenden Anzahl solcher Datenelemente kristallisieren sich Erkenntnisse heraus. ${ }^{13}$ Es stellt sich die Frage, wie viele Daten genügen. Insgesamt ist der Datensatz, den ich durch das oben beschrieben Vorgehen generieren konnte, im Umfang klein geblieben. Es wurde nicht der Punkt der Sättigung erreicht, der es mir erlauben würde, die eingangs gestellte Forschungsfrage zu beantworten, was bedeutet, dass ich die Frage dem bestehenden Datensatz anpassen muss.

Ein flexibler Umgang mit dem Forschungsdesign ist nicht ungewöhnlich und ein Charakteristikum ethnographischer Vorgehensweise. Das Problem, das sich stellt, ist vielmehr ein Problem des Kontexts. ${ }^{14}$ Für eine Mikrountersuchung, also eine Untersuchung eines spezifischen lokalen Kontexts und der Alltagspraktiken einzelner Familien, reicht meine Datenlage nur bedingt aus. Die Frage aber, wie ein Makrokontext, der die weitere Gesellschaft mit einbezieht, zu definieren sei und wie die notwendigen Daten hierfür zu generieren wären, ist nicht einfach zu beantworten. Martyn Hammersley argumentiert beispielsweise, dass als erstes die Frage $\mathrm{zu}$ beantworten ist, ob es sich um einen erweiterten Kontext handelt, der durch Forschung entdeckt wird, oder ob es ein vom Analysten konstruierter ist. ${ }^{15}$ Sie führt weiter aus, dass sich Schwierigkeiten ergeben bei der Eingrenzung des erweiterten Kontexts und der Situierung des Lokalen darin. Die Wahl dieses Kontexts sei letztlich in dem Sinne zufällig, dass verschiedene Perspektiven eingenommen werden können und jeder Forschungskontext in sich partiell bleibt. ${ }^{16}$

Im hier beschriebenen Projekt wurde die Erweiterung des Kontexts auf Grund der Datenlage zu einer Notwendigkeit. Die Daten selbst führten zu einer Verschiebung des Fokus und liessen den Alltag der Nomaden praktisch zur Nebensache werden. Die Mobilitäten der Nomaden konnten nicht genügend im Kontext der täglichen Aktivitäten und Arbeiten auf den Weidegebieten untersucht werden, sondern fast ausschliesslich in Bezug auf ihre Interaktion mit den Ortschaften und Städten, die Nomaden aus verschiedenen Gründen von Zeit zu Zeit aufsuchen. Der Kontext, der sich daraus ergibt, ist nur bedingt ein konstruierter. Er bettet die sich verändernde Mobilität der Nomaden in Prozesse der Modernisierung und Urbanisierung ein. Diese tragen massgeblich zu Veränderungen der nomadischen Gesellschaft bei und werden kontrovers diskutiert. ${ }^{17}$ Damit ordnet sich das Forschungsprojekt einem gesellschafts- und entwicklungspolitisch hoch aktuellen Kontext zu, den ich, um eine Forschungsbewilligung erwirken $\mathrm{zu}$ können, ursprünglich zu umgehen suchte.

13 Heimer 2006: 64.

14 Hammersley 2006.

15 Hammersley 2006: 6.

16 Hammersley 2006: 7-8; siehe auch Strathern 2004.

17 Bauer und Childs 2008; Kerven 2005. 
Die Makroebene des erweiterten Kontexts erarbeite ich durch ein methodisches Vorgehen, das erneut Fragen aufwirft. Meine Daten aus der jüngsten Feldforschung erlauben mir eine partielle Einsicht in neue und veränderte Mobilitäten von Nomaden. Diese setze ich einerseits in einen historischen Kontext, indem ich Rückgriff nehme auf Daten und Beobachtungen, die ich in früheren Jahren und in zum Teil anderen Arbeits- und Forschungskontexten generiert habe ${ }^{18}$. Andererseits erarbeite ich anhand der bestehenden Forschung und anhand von Dokumenten aus China und der unmittelbaren Forschungsregion die Entwicklung der Infrastruktur und ihre Konsequenzen in Bezug auf Governmentalität, die in der vom Staat betriebenen Entwicklungspolitik zum Ausdruck kommt. Die Frage, die sich mir stellt, ist, wie mit diesen verschieden gearteten Daten umzugehen ist.

Im neu gestalteten Dissertationsprojekt verfolge ich nun ein historiographisches Vorgehen, um die gegenwärtige Situation motorisierter Mobilität und mobiler Telekommunikation zu verstehen. In diesen Rahmen werden empirische Daten aus früheren Forschungsperioden (1999-2009) eingebettet, und die Frage stellt sich, wie mit ihnen umzugehen ist. Zusätzlich zur historischen Einordnung müssen die Daten sorgfältig gesichtet und darauf geprüft werden, ob sie einem allgemeinen ethischen Forschungsanspruch genügen. Dabei sind meine damalige Subjektposition und die Art, wie die Daten generiert wurden, zu reflektieren. Beispielsweise habe ich für einige Jahre als Projektmitarbeiterin für eine internationale Nicht-Regierungsorganisation gearbeitet und in dieser Zeit ethnographische Beschreibungen der Projektlokalitäten erstellt. Wie Mette Hansen (2006) gezeigt hat, treten Ethnographen - ob Anthropologen oder Personen der Entwicklungszusammenarbeit - ungewollt in die Fussstapfen der kommunistischen Partei und ihrer Praxis der soziologischen Befragungen von Menschen durch Institutionen des Staates. Das hat seine eigene Problematik und muss bei der Auswertung des Datenmaterials in die Analyse einbezogen werden. ${ }^{19}$ Es kann aber auch die Konsequenz haben, dass Menschen davon ausgehen, dass Befragungen Eingang in Berichterstattungen offiziellen Charakters finden. Dieser Freiraum eines impliziten Einverständnisses zur Verwendung von Informationen kann vorsichtig ausgeschöpft werden.

Nicht nur Daten aus früheren Forschungs- und Lebensperioden müssen auf meine damaligen Subjektpositionen reflektiert werden. Dies ist auch von zentra-

18 Ich habe von 1999 bis 2009 in Sichuan gelebt. In dieser Zeit habe ich in wechselnden Rollen Beobachtungen festgehalten und Gesprächsnotizen gemacht. Ich war einige Jahre Sprachstudentin, dann Forschende für meine Masterthese der Sozialanthropologie und anschliessend für unabhängige Forschungsarbeiten, und schliesslich war ich Programmverantwortliche für eine internationale NGO.

19 Hansen 2006: 82. 
ler Wichtigkeit bezüglich der von mir durchgeführten Guerilla-Feldforschung. In öffentlichen Räumen von Strassen, Restaurants, Teehäusern positionierte ich mich als Reisende und als Besucherin von alten Bekannten und von Freunden. Da ich weder Aufnahmegerät noch Notizblock benutzte, ${ }^{20}$ waren Interaktionen frei von Einschränkungen und Hemmungen aufgrund einer Rolle, die ein Gegenüber in einer Interviewsituation einnehmen kann. Die Schwierigkeit besteht vielmehr darin, dass aus ethischen Überlegungen eine Abgrenzung vorgenommen werden muss zwischen Gesprächen, in denen sich beide Parteien meiner Rolle bewusst waren und aus diesem Grund Informationen weitergegeben wurden, und rein informellen, freundschaftlichen Gesprächen. Diese Unterscheidung ist nicht in jedem Fall eindeutig. Der Umgang mit den daraus resultierenden ethischen Dilemmas muss schlussendlich der Urteilsfähigkeit des Forschenden überlassen werden. $^{21}$

Diese Urteilsfähigkeit muss auch zu Rate gezogen werden in Bezug auf Beobachtungen und Gespräche, die sich aus meiner Rolle als unbekannte, ausländische Reisende ergaben. Man kann sie als verdeckte Forschung betrachten. Eine solche wirft ethische Fragen in einem ähnlichen Ausmass auf wie die Grundsatzfrage, ob Forschung ohne Bewilligung vertretbar ist. Meine Entscheidung, beides zuzulassen, beruht darauf, dass es sich nicht um eine absichtliche Täuschung handelte und dass diese Art der Forschung sich auf Beobachtungen beschränkt, die weder den Persönlichkeitsschutz und die Anonymität der Beobachteten verletzen, noch Situationen beschreiben, die moralisch oder politisch für die betroffenen Personen kontrovers sein könnten. ${ }^{22}$ Deshalb sehe ich es als gerechtfertigt an, Daten aus nicht deklarierten Beobachtungen im öffentlichen Raum für die Forschung zu verwenden.

Ich halte fest, dass Guerilla-Feldforschung die Ethnographin in einem grossen Mass dazu verpflichtet, auch im Nachgang zur Forschung im Feld sich damit auseinanderzusetzen, wie Daten generiert wurden, welche Rolle der Kontext und die Person des Ethnographen dabei spielten, und wie verantwortungsvoll mit den

20 Wie Candice Cornet bemerkt, ist es in China ohne Bewilligung unmöglich, Interviews, die per Aufnahmegerät oder Notizblock aufgezeichnet werden, durchzuführen (Cornet 2010: 137). Man mag sich zu Recht die Frage nach dem wissenschaftlichen Anspruch an die Datenerhebung stellen und fragen, wie zuverlässig die generierten Daten sind. Ich verweise auf die ethnographische Praxis, welche die Person des Ethnographen in der Generierung und Interpretation der Daten impliziert sieht, unabhängig davon, ob ein Gespräch während oder nach der Durchführung aufgezeichnet wurde. Fabian (1990) beispielsweise zeigt auf, wie das Performative, also die Person des Ethnographen, dem Informativen, also den generierten Daten, gegenüber stehen. Er weist darauf hin, dass ersteres oft unterbewertet wird.

21 Wood 2006: 374.

22 Siehe auch Davies 1999: 53-58. 
Daten und den Gesprächspartnern, die zu einem grossen Teil zur Datengenerierung beigetragen haben, umzugehen ist. Dies sind grundsätzliche Vorgaben an qualitativ Forschende. Der ethische Anspruch bleibt insgesamt gleich, ist aber wesentlich akzentuierter. Es ist ein Zeichen von reflexivem Nachdenken, wenn in der Guerilla-Feldforschung die Messlatte hoch gelegt und das Vorgehen transparent dargestellt wird.

\section{Wieviel Transparenz? - Chancen und Grenzen der Guerilla-Feldforschung}

Ich habe in diesem Aufsatz darzustellen versucht, unter welchen komplexen Voraussetzungen ich meine Feldforschung durchführte und welche Art von Daten ich fähig war zu generieren. Ich habe damit Einblick gegeben in mein konstantes Fragen, ob Guerilla-Feldforschung legitim ist, inwiefern ich Freunde und Bekannte in die Datengeneration involvieren darf und ob es ethisch vertretbar ist, dieses Beobachtungsprotokoll und jene Gesprächsnotiz für mein Forschungsprojekt zu verwenden. Indem ich den Prozess der Forschung transparent mache, finde ich einen Umgang mit der Besorgnis, welche das untenstehende Zitat zum Ausdruck bringt.

One reason for glossing over problems encountered in fieldwork is no doubt a fear that the validity of our entire research will be jeopardized if we go public with those deviations from ideal methodological standards we are forced to make in the field“ ${ }^{23}$

Statt Probleme zu verdecken, entschied ich mich, sie zum Thema zu machen. Die hier ausgeführte Methode der Guerilla-Feldforschung zeigt auf, welche Entscheidungen ich getroffen habe. Ich möchte damit zu weiteren Diskussionen über den Umgang mit dem Unordentlichen und Unvorhergesehenen von Forschung im restriktiven Kontext anregen und zum Nachdenken über die manchmal unvorhersehbaren Konsequenzen ethnographischen Vorgehens.

Unvorhergesehenes kann ein neues Fenster der Erkenntnis öffnen. Obschon sich die Forschung nicht so entwickelt hat wie ursprünglich geplant, haben sich durch die Einschränkungen während der Feldforschung und die dadurch veränderte Datenlage neue, unerwartete Einsichten ergeben. Weil es nicht anders machbar war, habe ich viele Stunden an öffentlichen Plätzen und in Teehäu-

23 Heimer und Thøgersen 2006: 2. 
sern in nomadischen Gebieten verbracht. Das heisst, dass ich zwar nur sehr beschränkt den Alltag auf den Weiden der Nomaden miterlebt habe, dafür aber viel Zeit dort verbracht habe, wo Nomaden aufgrund ihrer technisierten Mobilität vermehrt hingehen. Dort haben sich mir neue Fragen und Zusammenhänge eröffnet in Bezug auf Handlungsräume von Nomaden und einer möglichen Ausgestaltung von öffentlichem und privatem Raum und der Rolle, die Mobilität dabei spielt. Durch ein Teilnehmen am Alltäglichen in den öffentlichen Räumen, durch Beobachten und durch ein in Beziehung treten mit Menschen, Dingen und deren Umfeld wurde ein Prozess ausgelöst, der anfänglich eher fluid und unbestimmt

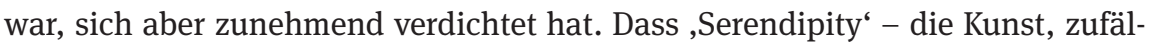
lige Beobachtungen mit intuitivem Reflektieren zu verbinden - zu unerwarteten und manchmal auch brauchbaren Ergebnissen führen kann, wurde bereits als wichtiger Aspekt ethnographischen Arbeitens vorgeschlagen. ${ }^{24}$ Im Falle dieses Dissertationsprojekts hat sich der Schwerpunkt der Forschungsfrage verschoben. Durch einen sorgfältigen Umgang mit der Vielfalt an Beobachtungen, Interaktionen mit Gesprächspartnern und zufälligen Begegnungen, wird schlussendlich aber ein Bild, bzw. eine Monographie entstehen, die zur Mobilitäts- und Pastoralismusforschung in Asien beitragen kann.

\section{Bibliographie}

Arya, S. (2011): „Infrastructure development and Chinese war waging capabilities in Tibet.“ Journal of Defense Studies 5.3: 81-97.

Association, American Anthropological (2012): „Statement on ethics: Principles of professional responsibilities.“ Arlington, VA: American Anthropological Association.

Bauer, K. / Childs, G. (2008): „Demographics, development, and the environment in Tibetan areas." Journal of the International Association of Tibetan Studies 4: 1-8.

Billo, E. / Hiemstra, N. (2012): „Mediating messiness: Expanding ideas of flexibility, reflexivity, and embodiment in fieldwork. “ Gender, Place \& Culture 20.3: 313-328.

Bonnin, C. (2010): „Navigating fieldwork politics, practicalities and ethics in the upland borderlands of northern Vietnam."Asia Pacific Viewpoint 51.2: 179-192.

Bryman, A. (2001): Social research methods. Oxford: Oxford University Press.

Cornet, C. (2010): „Fieldwork among the Dong national minority in Guizhou, China: Practicalities, obstacles and challenges." Asia Pacific Viewpoint 51.2: 135-147.

Davies, C.A. (1999): Reflexive ethnography: A guide to researching selves and others. Oxon/ New York: Routledge.

de Certeau, M. (1988): The practice of everyday life. Berkeley: University of California Press. Démurger, S. (2001): „Infrastructure development and economic growth: An explanation for regional disparities in China?" Journal of Comparative Economics 29.1: 95-117.

24 Rivoal und Salazar 2013. 
Fabian, J. (1990): Power and performance: Ethnographic explorations through proverbial wisdom and theater in Shaba, Zaire. Madison: Wisconsin University Press

Fabian, J. (2001): Anthropology with an attitude: Critical essays. Stanford: Stanford University Press.

Flower, J. M. (2004): „A road is made: Roads, temples, and historical memory in Ya'an County, Sichuan." The Journal of Asian Studies 63.3: 649-685.

Garver, J. W. (2006): „Development of China's overland transportation links with Central, South-West and South Asia.“ The China Quarterly 185: 1-22.

Geertz, C. (1973): The Interpretation of Cultures. New York: Basic Books.

Gold, T.B. (1989): „Guerilla interviewing among the Getihu.“ In: Unofficial China: Popular Culture and Thought in the People's Republic. Hrsg. von P. Link, R. Madsen, und P. Pickowicz. Boulder: Westview Press, 175-192.

Hammersley, M. (2006): „Ethnography: problems and prospects.“ Ethnography and Education 1.1: 3-14.

Hansen, M.H. (2006): „In the footsteps of the Communist party: Dilemmas and strategies.“ In: Doing Fieldwork in China. Hrsg. von M. Heimer und S. Thøgersen. Honolulu: University of Hawai'i Press, 81-95.

Heimer, M. (2006): „Field sites, research design and type of findings.“ In: Doing Fieldwork in China. Hrsg. von M. Heimer und S. Thøgersen. Honolulu: University of Hawai’i Press, $58-80$.

Heimer, M. / Thøgersen, S. (Hrsg.) (2006): Doing Fieldwork in China: University of Hawai'i Press.

Kerven, C. (Hrsg.) (2005): Nomadic Peoples 9.1-2 Speical issue: Pastoralists in Post-socialist Asia.

Rivoal, Isabelle / Salazar, Noel B. (2013): „Contemporary ethnographic practice and the value of serendipity.“ Social Anthropology 21.2: 178-185.

Sautman, B. (2006): „Colonialism, Genocide, and Tibet.“Asian Ethnicity 7.3: 243-265.

Schüller, M. / Schüler-Zhou, Y. / Peterskovsky, L. (2010): Chinas Telekommunikationsunternehmen drängen an die Weltspitze. GIGA Focus Asien 12 : online <http://www.gigahamburg.de/de/system/files/publications/gf_asien_1012.pdf>

Strathern, M. (2004): Partial Connections. Walnut Creek, CA: AltaMira Press.

Wang, Q. (2007): „Progress of transportation development in China.“Journal of Transportation, System Engineering and Information Technology 7.1: 1-11.

Wood, E. J. (2006): „The ethical challenges of field research in conflict zones.“ Qualitative Sociology 29.3: 373-386.

Yeh, E. T. (2006): „,An open Lhasa welcomes you“: Disciplining the researcher in Tibet.“ In: Doing fieldwork in China. Hrsg. von M. Heimer und S. Thøgersen. Honolulu: University of Hawai'i Press, 96-109. 